This document is confidential and is proprietary to the American Chemical Society and its authors. Do not copy or disclose without written permission. If you have received this item in error, notify the sender and delete all copies.

\title{
Charge transfer in single chains of a donor-acceptor conjugated tri-block copolymer
}

\begin{tabular}{|r|l|}
\hline Journal: & The Journal of Physical Chemistry \\
\hline Manuscript ID: & Draft \\
\hline Danuscript Type: & Special Issue Article \\
\hline Complete List of Authors: & $\begin{array}{l}\text { Hooley, Emma; University of Melbourne, School of Chemistry } \\
\text { Jones, David; University of Melbourne, School of Chemistry } \\
\text { Greenham, Neil; University of Cambridge, Department of Physics } \\
\text { Ghiggino, Kenneth; University of Melbourne, Chemistry } \\
\text { Bell, Toby; Monash University, School of Chemistry }\end{array}$ \\
\hline
\end{tabular}




\title{
Charge transfer in single chains of a donor-acceptor
} conjugated tri-block copolymer

\author{
Emma N. Hooley, ${ }^{A}$ David J. Jones, ${ }^{A}$ Neil C. Greenham, ${ }^{B}$ \\ Kenneth P. Ghiggino, ${ }^{A^{*}}$ Toby D. M. Bell, ${ }^{C^{*}}$ \\ ${ }^{\text {A }}$ School of Chemistry, University of Melbourne, Victoria 3010, Australia \\ ${ }^{\mathrm{B}}$ Cavendish Laboratory, University of Cambridge, J. J. Thomson Avenue, Cambridge CB3 OHE, United \\ Kingdom \\ ${ }^{\mathrm{C}}$ School of Chemistry, Monash University, Clayton, Victoria 3800, Australia
}

\begin{abstract}
AUTHOR EMAIL ADDRESS toby.bell@monash.edu; ghiggino@unimelb.edu.au
RECEIVED DATE (to be automatically inserted after your manuscript is accepted if required according to the journal that you are submitting your paper to)

TITLE RUNNING HEAD Charge transfer in a tri-block copolymer.

CORRESPONDING AUTHORS FOOTNOTE TDMB: Tel +61 39905 4566, Fax +61 39905 4597;

KPG: Tel. +61 38344 8939, Fax +61393475180
\end{abstract}




\begin{abstract}
:
The photophysics of a conjugated tri-block copolymer comprising poly(9,9-dioctylfluorene-co-bisN,N'-(4-methylphenyl)-bis-N,N'-phenyl-1,4-phenylenediamine (PFM) electron donor and poly-(4-(9,9dioctyl-9H-fluoren-2-yl)benzo[c][1,2,5]-thiadiazole) (F8BT) electron acceptor blocks has been studied in solution, in films and as single chains. While an additional long-wavelength emission apparent in neat films of the copolymer is attributed to inter-chain exciplex formation, no such long-wavelength emission is apparent in solution or from single molecules. However, in these cases, time-resolved fluorescence measurements indicate the presence of a delayed fluorescence. The kinetics of the delayed emission can be interpreted in terms of an equilibrium between a locally-excited and a charge-transfer state at the interface of the copolymer block components. Rate constants and thermodynamic quantities associated with these processes have been evaluated. The single molecule results allow the assignment of an intramolecular charge-transfer state in an isolated conjugated block copolymer chain.
\end{abstract}

\footnotetext{
KEYWORDS Conjugated polymer, block copolymer, single molecule fluorescence, charge transfer, delayed fluorescence, exciplex.
} 


\section{Introduction:}

Polymers containing sequences of $\pi$-conjugation along the polymer backbone have attracted considerable interest, since their combination of optical and electronic properties lends them to applications in organic photovoltaics ${ }^{1,2}$ and organic light-emitting diodes. ${ }^{3}$ Following photo-excitation these 'conjugated polymers' can act as electron donors in the presence of other small-molecule or polymer electron acceptors and this provides the basis for the use of these materials in organic solar cells. $^{4,5}$ Photo-excitation at first creates a bound electron-hole pair or exciton that can migrate over short distances in the conjugated polymer. To achieve photo-current generation, the exciton needs to encounter a suitable electron acceptor, to ultimately yield complete separation of the charges that can then migrate independently to the appropriate electrode. Thus efficient solar cell performance requires both effective charge transfer and separation at the interface between the donor and acceptor species as well as a continuous pathway for the unbound charges to travel to the device electrodes. Bulk heterojunction solar cells made up of blends of the donor and acceptor materials go some way to solving these problems, however careful control over the morphology of the film components is necessary. ${ }^{6,7}$

In order to impose an element of control over the morphology of the active layer in organic photovoltaic devices, block copolymers consisting of electron donor and acceptor blocks have been investigated $^{8-11}$ and show some promise in optimizing charge separation. As each polymer chain contains a donor-acceptor interface, intramolecular charge transfer (CT) can occur. Including donoracceptor interfaces within a single polymer chain ensures close contact between donor and acceptor blocks, and reduces the influence of blend morphology on the extent and position of the donor-acceptor interface. Utilizing self-assembly behavior, a structured environment can be provided for the separated, unbound charges to travel freely to the electrodes.

Both intramolecular CT and intermolecular CT are possible when an appropriate lower energy CT state (with respect to the locally excited state) exists and can be populated. Non-radiative recombination is a common fate of CT states, however radiative relaxation resulting in emission from the CT state is also possible. Polymer blend ${ }^{12,13}$ and small molecule studies ${ }^{14,15}$ have shown that, given an appropriate 
energy arrangement of electronic states involved, photo-induced electron-transfer processes can result in an equilibrium between the locally-excited (LE) singlet state and the CT state. The position of the equilibrium is often dependent on the local environment which can control the energetics. ${ }^{16}$ While the CT state has been studied in donor-acceptor conjugated polymers, ${ }^{17-19}$ it has not been reported at the single-chain level. Back electron transfer from fully charge separated species can lead to re-formation of the ground state, however, when the energy levels are appropriate and the LE state and CT state are in equilibrium, a delayed fluorescence can occur facilitated by thermal back transfer from the CT state to regenerate the LE state.

In this work, we report on the photophysics of a donor-acceptor tri-block copolymer PFM-F8BTPFM, consisting of electron donor PFM (poly(9,9-dioctylfluorene-co-bis-N,N'-(4-methylphenyl)-bisN,N'-phenyl-1,4-phenylenediamine)) and electron acceptor F8BT (poly[(9,9-di-n-octylfluorenyl-2,7diyl)-alt-(benzo[2,1,3]thiadiazol-4,8-diyl)), (Chart 1). This donor-acceptor block copolymer has been used as the active layer in photovoltaic devices ${ }^{20}$ where it outperformed a simple $2: 1$ blend of PFM:F8BT by a factor of three in terms of power conversion efficiency of the devices. In this present work, the observation of a delayed fluorescence component in solution, neat film, and in single chains is attributed to the presence of an intrachain charge-transfer state in the block copolymer. 
Chart 1. The chemical structure of the PFM-F8BT-PFM triblock copolymer and PFM and F8BT homopolymers.
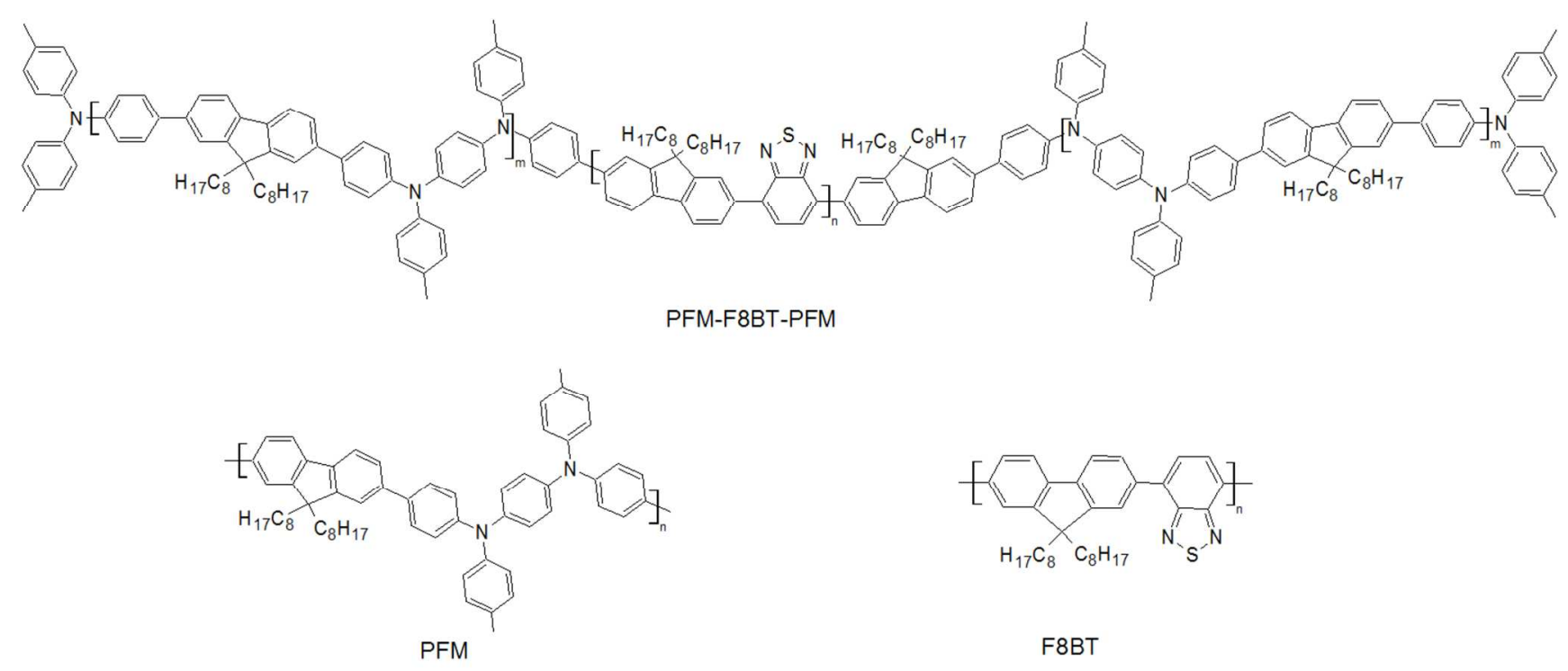

\section{Experimental:}

A central poly-(4-(9,9-dioctyl-9H-fluoren-2-yl)benzo[c][1,2,5]-thiadiazole) (F8BT) electronaccepting block was synthesised by Suzuki condensation, followed by endcapping with trimethylsilylpheny groups and reaction with $\mathrm{ICl}$. The block size was controlled by monomer ratio followed by removal of low molecular weight oligomers via soxhlet extraction with ethyl acetate leaving a polymer with $\left(\mathrm{M}_{n}=21,200, \mathrm{M}_{w}=32,000, \mathrm{M}_{w} / \mathrm{M}_{n}=1.51\right)$. Chain extension with N1-(4-(7-(4iodophenyl)-9,9-dioctyl-9H-fluoren-2-yl)phenyl)- $N^{1}, N^{4}$-di-p-tolylbenzene-1,4-diamine under Buchwald-Hartwig conditions, followed by endcapping with bromotoluene, resulted in the formation of the triblock copolymer PFM-F8BT-PFM. The triblock copolymer had a PFM:F8BT weight ratio of 1:1 $\left(M_{n}=43,500, M_{w}=56,400, M_{w} / M_{n}=1.30\right)$. Synthetic polymerization and characterization details and photovoltaic device performance of this block copolymer have been published elsewhere. ${ }^{20,21}$

Absorption spectra were recorded using a Cary 50 Bio UV-Vis spectrophotometer (Agilent) with solvent baseline subtraction and a Cary Eclipse Fluorimeter (Agilent) was used to measure solution emission spectra. Bulk samples were prepared using spectroscopic grade solvents (Aldrich) which were 
used as received. Fluorescence quantum yields in solution were determined from emission spectra corrected for the wavelength dependence of the detector efficiency by comparing the emission of PFMF8BT-PFM against the standard disodium fluorescein in ethanol $\left(\Phi_{\mathrm{fl}}=0.79\right) .{ }^{22}$ Degassed solutions where the maximum absorbance was kept below 0.1 were used to avoid oxygen quenching and inner filter effects.

Fluorescence lifetimes were determined by the method of time-correlated single photon counting (TCSPC). Full details of the experimental set-up and data analysis are published elsewhere. ${ }^{23}$ Briefly, excitation was provided by a 'white light' super-continuum fiber laser source (Fianium SC-400-4-pp) operated at $5 \mathrm{MHz}$ and $488 \pm 5 \mathrm{~nm}$ light selected using a narrow bandpass filter (Chroma). Fluorescence was collected at $90^{\circ}$ through a monochromator (Digikrom DK480) and focused onto an avalanche photodiode (APD) (IdQuantique Id-100). Data collection and photon arrival timing was achieved using a photon counting module (PicoQuant PicoHarp300) and transferred to a PC. Decay curves were analyzed by a least squares fitting method based on the Levenberg-Marquardt algorithm using the TRFA Global Analysis program (Scientific Software Technologies Centre) taking into account the instrument response function (IRF). For film measurements, samples were prepared on clean glass coverslips and mounted on a confocal, inverted fluorescence microscope (Olympus IX71). Laser light was focused through a high numerical aperture objective (Olympus PSPlanApo 100x/1.4 NA) and fluorescence was collected through the same objective, separated from the excitation light by a dichroic filter (Chroma) and focused onto an APD (PicoQuant TauSPAD). Instrument response functions (IRF) were measured from a scattering solution or from a clean, blank coverslip. Emission spectra from films were measured using the confocal microscope with the fluorescence emission directed through a spectrograph (Princeton Instruments Acton SP2150) and onto an electron multiplying CCD camera (Princeton Instruments ProEm512). 
For single molecule spectroscopy, samples were diluted to $\sim 1 \times 10^{-9} \mathrm{~mol}_{\mathrm{dm}}{ }^{-3}$ of polymer by serial dilution in $\mathrm{CHCl}_{3}$ (Aldrich) with the final dilution into a $\sim 0.5 \%$ (w/w) solution of poly(methyl methacrylate) (PMMA) (Aldrich) in $\mathrm{CHCl}_{3}$. Solutions were spin-coated (Specialty Coating Systems P6700) onto a clean glass coverslips to produce a thin polymer film, approximately $100-200 \mathrm{~nm}$ thick. Confocal single molecule measurements were performed either on the microscope described above with the sample mounted on a $x-y$ piezo stage, (Physik Instrumente P-517), or on a similar microscope described elsewhere. ${ }^{24}$ A clean, blank slide was used to measure the IRF for analysis of single molecule fluorescence lifetimes. Single molecule trajectories and fluorescence lifetimes were determined using the BIFL software package (Scientific Software Technologies Center). Further details of the instrument, data collection and analysis procedures have been outlined previously. ${ }^{25}$ Widefield single molecule images were recorded on an inverted single molecule microscope described previously. ${ }^{26}$ Defocussed images were obtained by moving the objective towards the sample by $\sim 1$ um revealing the angular distribution of emitted light intensity. ${ }^{27,28}$ The patterns observed are a function of the emission dipole orientation and can be related to molecular orientation as well as indicate whether the image is from a single dipole emitter. The defocused image patterns were matched to theoretical patterns calculated as described elsewhere. ${ }^{29,30}$

\section{Results and discussion:}

Donor-acceptor block copolymers must have appropriate energy levels and redox properties to allow for effective charge transfer; in this case from the electron donor PFM block, to the F8BT acceptor block. $^{31}$ Figure 1 shows the absorption spectrum of the block copolymer in toluene solution and it features three distinct but overlapping absorption bands with wavelength maxima at approximately $330 \mathrm{~nm}, 390 \mathrm{~nm}$ and $470 \mathrm{~nm}$. The shape of the PFM-F8BT-PFM absorbance spectrum can be described by the sum of the absorbance spectra of the two homopolymer blocks that comprise the copolymer (SI Figure 1) with assignment of the bands as follows: the $300 \mathrm{~nm}$ and $470 \mathrm{~nm}$ bands are associated with 
absorption by the F8BT block and the $390 \mathrm{~nm}$ band with PFM absorption. The solution absorbance spectra of the homopolymers correspond well to spectra reported elsewhere. ${ }^{32,33}$

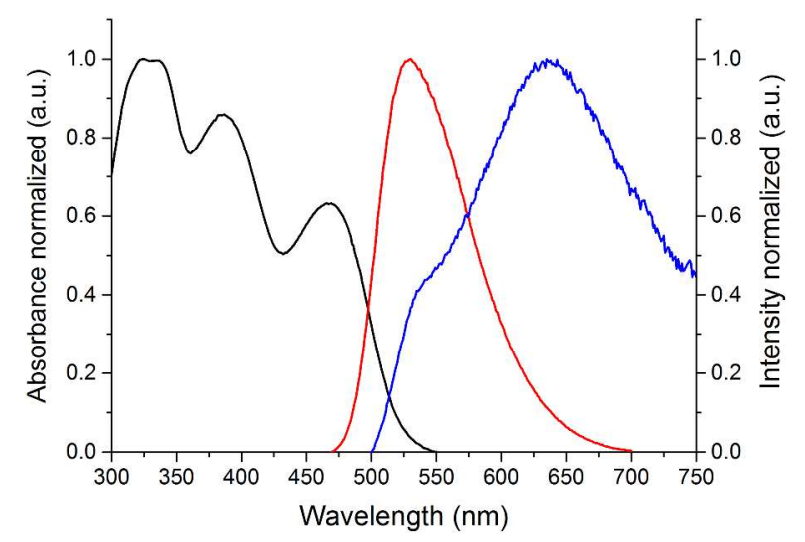

Figure 1. Absorption and emission spectra of PFM-F8BT-PFM. The absorption spectrum is measured in toluene and emission spectra are measured in toluene (red), and as a neat film (blue), $\left(\lambda_{\mathrm{exc}}=470 \mathrm{~nm}\right)$.

Fluorescence emission from all of PFM, F8BT and PFM-F8BT-PFM shows a shift to lower energy with increasing solvent polarity (SI Figure 2 and SI Table 1). F8BT undergoes a red-shift of $37 \mathrm{~nm}$ $\left(1300 \mathrm{~cm}^{-1}\right)$, from $512 \mathrm{~nm}$ in cyclohexane to $549 \mathrm{~nm}$ in benzonitrile, whereas for PFM, a much larger shift of $95 \mathrm{~nm}\left(4100 \mathrm{~cm}^{-1}\right)$ occurs on going from cyclohexane to dichloromethane (PFM not being soluble in benzonitrile). The shape of the emission spectrum also changes with the polarity of the solvent. PFM emission in cyclohexane is narrow and shows some vibronic structure, but becomes increasingly broad and red-shifted as the solvent polarity increases. This large solvatochromic effect is consistent with reports of other polyfluorenes. ${ }^{19,34}$ PFM-F8BT-PFM shows some difference in solvatochromism depending on the excitation wavelength. When the PFM block is excited at $380 \mathrm{~nm}$, (the selectivity over F8BT at this wavelength is approximately 8:1) the PFM-F8BT-PFM copolymer shows mostly PFM-like emission in cyclohexane with some F8BT emission. The proportion of F8BT emission increases in toluene and in the more polar solvent $\mathrm{CHCl}_{3}$ primarily $\mathrm{F} 8 \mathrm{BT}$-like emission is observed as is the case when solvents of even higher polarity are used. When PFM-F8BT-PFM is 
excited at $460 \mathrm{~nm}$ (F8BT is excited almost exclusively at this wavelength), the emission spectrum is associated with F8BT, as expected.

Changing the polarity of the solvent also affects the fluorescence quantum yield of PFM-F8BT-PFM. In toluene, the polymer shows a fluorescence quantum yield of 0.38 , which drops significantly to 0.17 in $\mathrm{CHCl}_{3}$. The stabilization of an available charge-transfer state, ${ }^{35,36}$ which may form at the interface between donor and acceptor blocks, is the likely reason for the increase in the non-radiative decay in the more polar solvent leading to the decrease in the fluorescence quantum yield. Photo-induced absorption spectroscopy has shown some evidence of the formation of a charge transfer state following photoexcitation of the PFM block for the co-polymer, however, the transient spectra were dominated by triplet state transient absorptions. ${ }^{20}$

The emission spectrum of a neat PFM-F8BT-PFM film is also shown in Figure 1. The neat film shows a very different emission spectrum compared to solution spectra and comprises a broad, redshifted emission in the $600-700 \mathrm{~nm}$ range as well as some F8BT-like emission at $550 \mathrm{~nm}$. The redshifted emission is consistent with reports that PFM-F8BT-PFM forms an exciplex in the bulk state. ${ }^{20}$ The lack of red shifted emission in solution suggests that emissive exciplex formation requires close inter-chain interactions.

\section{Time-resolved measurements:}

Figure $2 \mathrm{a}$ shows the fluorescence decay profile of the homopolymer F8BT in toluene solution and neat film. In toluene, F8BT decays mono-exponentially with a fluorescence lifetime of 2.2 ns while in neat film its decay is more complex with a second, shorter-lifetime component present contributing the bulk of the light emitted. This suggests that in a solid film, environmental heterogeneity and inter-chain interactions affect the majority of the excited F8BT chromophores such that the fluorescence detected decays on a much shorter timescale. A small portion of the F8BT chromophores in the film, however, emit with a similar decay time (2.2 ns) as in solution. Figure $2 \mathrm{~b}$ shows the fluorescence decay of 
PFM-F8BT-PFM, excited at $488 \mathrm{~nm}$. Interestingly, in toluene, a bi-exponential decay function is required and in film, the decay requires the sum of four exponential terms to achieve satisfactory fitting, indicating that additional relaxation pathways, compared to F8BT, contribute to the observed emission in both solution and neat film. As seen for F8BT film, there is a fast sub-nanosecond component in neat film of the copolymer, attributable to inter-chain interactions. As mentioned above, there is the possibility for the locally excited state to form an excited state donor-acceptor complex (exciplex) with an adjacent chain where the interacting species meet the geometric requirements. This can occur in bulk films where PFM blocks can stack with the F8BT blocks from nearby chains. The longest lifetime in neat film $\left(\tau_{4}=19 \mathrm{~ns}\right)$ is attributed to these intermolecular states. The fitted parameters for all these samples are given in Table 1.
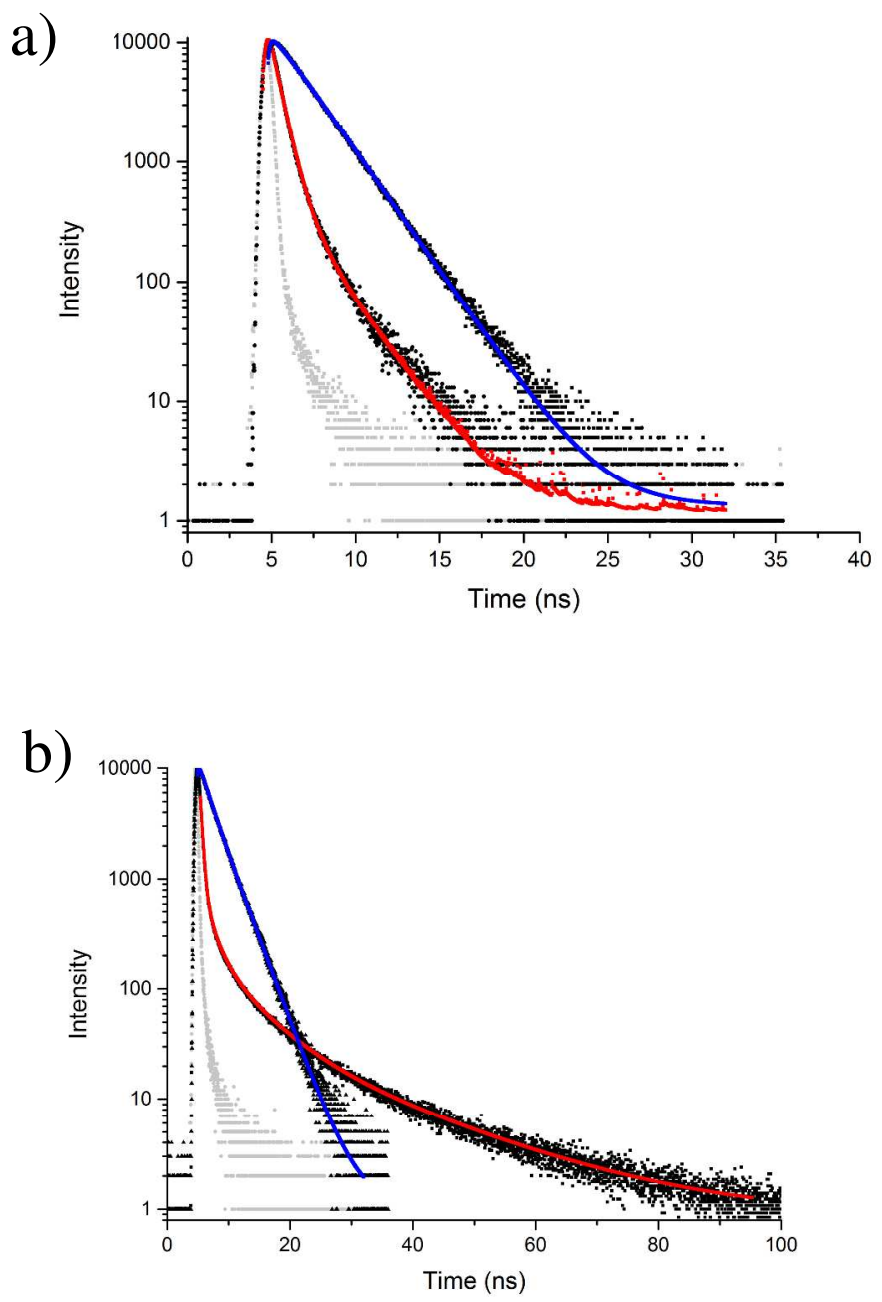
Figure 2. Fluorescence decays (black data points) and fitted functions (colored lines) of a) F8BT and b) PFM-F8BT-PFM, in toluene (blue) and neat film (red). Excitation was at $488 \mathrm{~nm}$ with emission detected at $560 \mathrm{~nm}$. The instrument response function is shown in grey.

Table 1. Fluorescence decay data for F8BT and PFM-F8BT-PFM in toluene solution and neat film.

\begin{tabular}{cccccccc}
\hline & \multicolumn{3}{c}{ F8BT } & & \multicolumn{3}{c}{ PFM-F8BT-PFM } \\
\cline { 2 - 3 } \cline { 5 - 7 } & $\tau_{1}(\mathrm{~ns})$ & $\tau_{2}(\mathrm{~ns})$ & & $\tau_{1}(\mathrm{~ns})$ & $\tau_{2}(\mathrm{~ns})$ & $\tau_{3}(\mathrm{~ns})$ & $\tau_{4}(\mathrm{~ns})$ \\
\hline Toluene & $2.2(100)^{a}$ & - & & $1.5(23)$ & $2.9(77)$ & - & - \\
Neat film & $0.59(84)$ & $2.2(16)$ & & $0.39(34)$ & $1.7(27)$ & $5.9(19)$ & $19(20)$ \\
\hline
\end{tabular}

In addition to the formation of excited state complexes, donor-acceptor copolymers are capable of intra-molecular charge transfer at the interface between the donor and acceptor blocks. ${ }^{12,31,35-38}$ The charge-transfer state for the closely related donor-acceptor pair, PFB and F8BT has been estimated at $1.93 \mathrm{eV}$, using the methods outlined by Janssen et al. ${ }^{35}$ The complex fluorescence decay kinetics observed in the present system suggests that a charge transfer state is also available and accessible from the locally excited (LE) state of F8BT. ${ }^{31}$ The usual fate of charge-transfer (CT) states is charge recombination to reform the ground state non-radiatively although it possible for this to be a radiative relaxation via charge-transfer emission. In rare circumstances where the energy levels are appropriately well matched and the LE and CT states suitably coupled, the LE-CT equilibrium can lead to a delayed fluorescence. Figure 3 shows the kinetic scheme for such a situation. An equilibrium is established between the LE state and the CT state and two fluorescence lifetimes are observed: a shorter fluorescence decay associated with quenching of the excited state by CT and a longer decay component arising following thermal regeneration of the LE state. When the CT state is non-emissive, any emission observed has the spectral characteristics of the LE state. For the tri-block copolymer under investigation here, the F8BT-like emission spectrum observed and the non-single exponential decay kinetics are 
consistent with a delayed fluorescence. In toluene solution, fluorescence emission is characteristic of F8BT and the two decay components can be associated with forward CT (1.5 ns) and reverse CT (thermal regeneration of LE) (2.9 ns), respectively. In neat film, there is the added complication of the much faster (sub ns) component due to interchain interactions as seen for F8BT alone. A delayed fluorescence is clearly observable with associated fluorescence components of 1.7 ns (forward CT) and 5.9 ns (reverse CT), respectively. Further evidence that emission is occurring from a state with characteristics similar to F8BT comes from Lippert-Mataga plots (SI Figure 3) of the steady state emission from PFM, F8BT and PFM-F8BT-PFM. The nature of the excited state of PFM is clearly different to that of F8BT whereas for the copolymer it is very similar to F8BT. If emission in the copolymer were from a CT state, a very different solvent polarity gradient would be expected to that of the F8BT state.

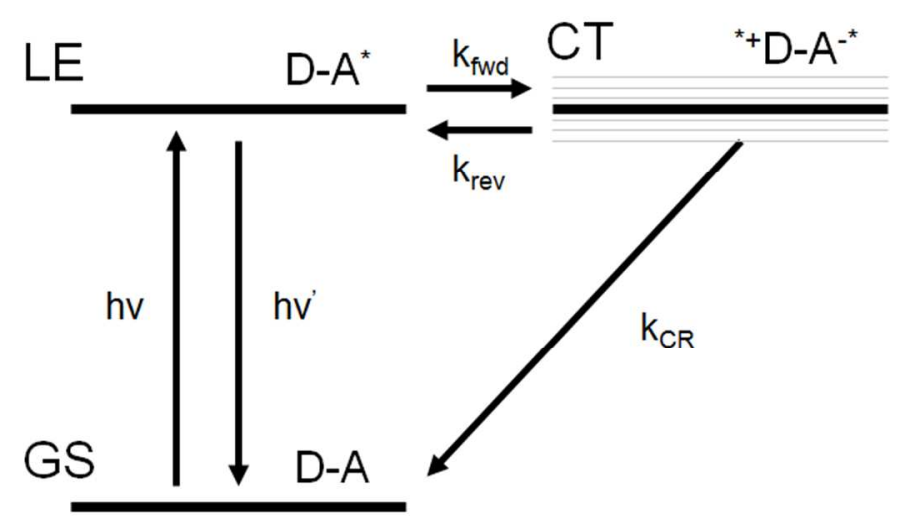

Figure 3. Kinetic scheme describing the interaction between the ground (GS), locally excited (LE) and charge transfer (CT) states for the donor-acceptor (D-A) block copolymer. The LE state is depicted with the acceptor excited since in the majority of these measurements, it is F8BT which is photoexcited. The fainter lines above and below the CT state indicate the variation of the energy of the CT state with polarity.

Based on the energy level scheme described in Figure 3, the rate constants and driving forces for the forward and reverse charge transfer processes can be calculated. ${ }^{39}$ The rate constants of forward and reverse charge transfer between the LE and CT states ( $k_{\mathrm{fwd}}$ and $k_{\mathrm{rev}}$, respectively) and the rate constant 
for charge recombination to the GS $\left(k_{\mathrm{CR}}\right)$ are calculated from the decay times $\left(\tau_{\mathrm{i}}\right.$ and $\left.\tau_{\mathrm{j}}\right)$ and preexponential factors $\left(a_{\mathrm{i}}\right.$ and $\left.a_{\mathrm{j}}\right)$ of the two decay components of the total fluorescence decay attributed to delayed fluorescence (i.e. those associated with the forward and reverse CT processes), and $\tau_{0}$, the fluorescence lifetime of the unquenched acceptor (details of the calculations are provided in the Supporting Information). $k_{\mathrm{fwd}}$ and $k_{\mathrm{rev}}$ in toluene are found to be $6.8 \times 10^{7} \mathrm{~s}^{-1}$ and $4.0 \times 10^{8} \mathrm{~s}^{-1}$, respectively. $k_{\mathrm{CR}}$ is slower than $k_{\mathrm{rev}}$ at $1.6 \times 10^{8} \mathrm{~s}^{-1}$, indicating that the major pathway for CT relaxation is back through the LE state and not via recombination to the GS. These rate constants give an equilibrium constant $\left(K_{\text {eq }}\right)$ between the LE and CT states of 0.17 and a driving force $\left(\Delta G_{\mathrm{CT}}\right)$ of +0.045 $\mathrm{eV}$ from the LE to the CT state. This shows that the equilibrium favors slightly the LE state and that the initial charge transfer process is energetically slightly unfavorable, but that the CT state is still accessible at room temperature. The closeness in energy of the LE and CT states implies that the energy of the CT state in toluene is approximately $2.5 \mathrm{eV}$, taking the energy of the LE state as that where the normalized absorption and emission spectra intersect $(503 \mathrm{~nm}$, see Figure 1). This is consistent with the value mentioned above for the CT state of PFB-F8BT which was obtained in ortho-dichlorobenzene. This solvent is moderately polar $\left(\varepsilon_{\mathrm{s}} \sim 10\right)$ and would lower the energy of the CT state compared to toluene. Delayed fluorescence is also apparent in neat film and $k_{\mathrm{fwd}}$ and $k_{\mathrm{rev}}$ are calculated to be $1.2 \times$ $10^{8} \mathrm{~s}^{-1}$ and $2.1 \times 10^{8} \mathrm{~s}^{-1}$ respectively, yielding a $K_{\text {eq }}$ of 0.58 and $\Delta G_{\mathrm{CT}}$ of $0.014 \mathrm{eV}$ indicating that CT is marginally more favored than in toluene. $k_{\mathrm{CR}}$, however, in the neat film $\left(3.4 \times 10^{7} \mathrm{~s}^{-1}\right)$ is considerably slower and less competitive than in solution, likely a result of the much more rigid nature of a neat film.

Table 2. Rate constants and related parameters derived for charge transfer in PFM-F8BT-PFM in solution and film (rate constants defined in Figure 3).

\begin{tabular}{lccccc}
\hline & $\begin{array}{c}k_{\mathrm{fwd}} \\
\left(10^{8} \mathrm{~s}^{-1}\right)\end{array}$ & $\begin{array}{c}k_{\mathrm{rev}} \\
\left(10^{8} \mathrm{~s}^{-1}\right)\end{array}$ & $\begin{array}{c}k_{\mathrm{CR}} \\
\left(10^{8} \mathrm{~s}^{-1}\right)\end{array}$ & $K_{\text {eq }}$ & $\begin{array}{c}\Delta \mathrm{G}_{\mathrm{CT}} \\
(\mathrm{eV})\end{array}$ \\
\hline Toluene & 0.7 & 4.0 & 1.6 & 0.17 & 0.045 \\
Neat film & 1.2 & 2.1 & 0.3 & 0.58 & 0.014 \\
\hline
\end{tabular}


Single molecule experiments:

Figure 4 shows two confocal fluorescence trajectories obtained from individual PFM-F8BT-PFM chains in PMMA. The intensity of both molecules fluctuates over time, with counts rates early in the trajectories as high as $5000 \mathrm{~s}^{-1}$ and $12000 \mathrm{~s}^{-1}$ for the two chains. Both molecules show good photostability emitting photons over minutes with the second molecule still emitting after 10 minutes of pulsed laser excitation. The fluorescence decay kinetics of the two molecules are strikingly different. The molecule shown in a) emits with mono-exponential decay kinetics while molecule b) is biexponential for the duration of its trajectory. These two molecules are essentially representative of the data set. Of the 149 PFM-F8BT-PFM chains measured, 137 showed either mono (72 molecules, 48\%) or bi-exponential (65 molecules, 44\%) kinetics for their entire trajectory, while the remaining 12 molecules $(8 \%)$ showed periods of each type of decay kinetics (at least 20000 photons or two adjacent data points). In contrast to this, single chains of the homopolymer F8BT (see SI Figure S4 for an example) show exclusively mono-exponential decays (103 molecules examined). 


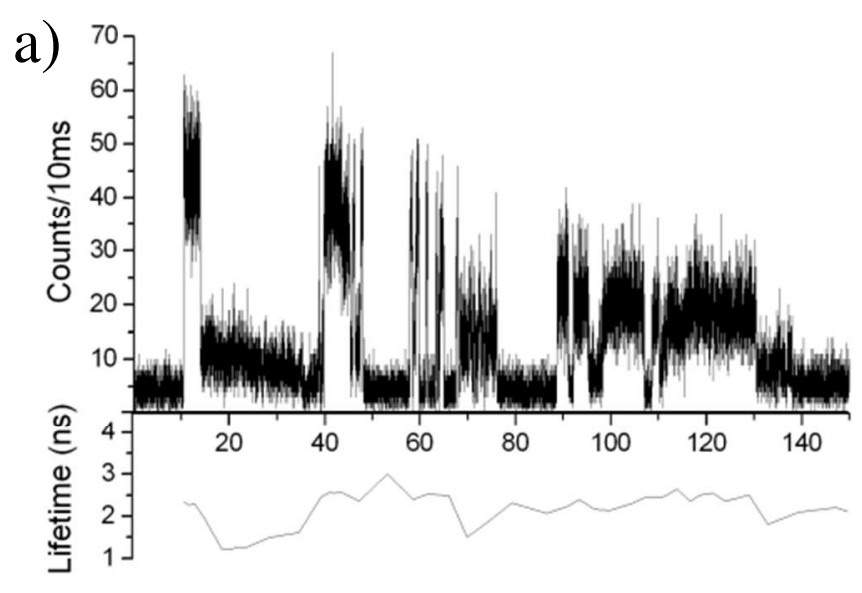

Time (s)

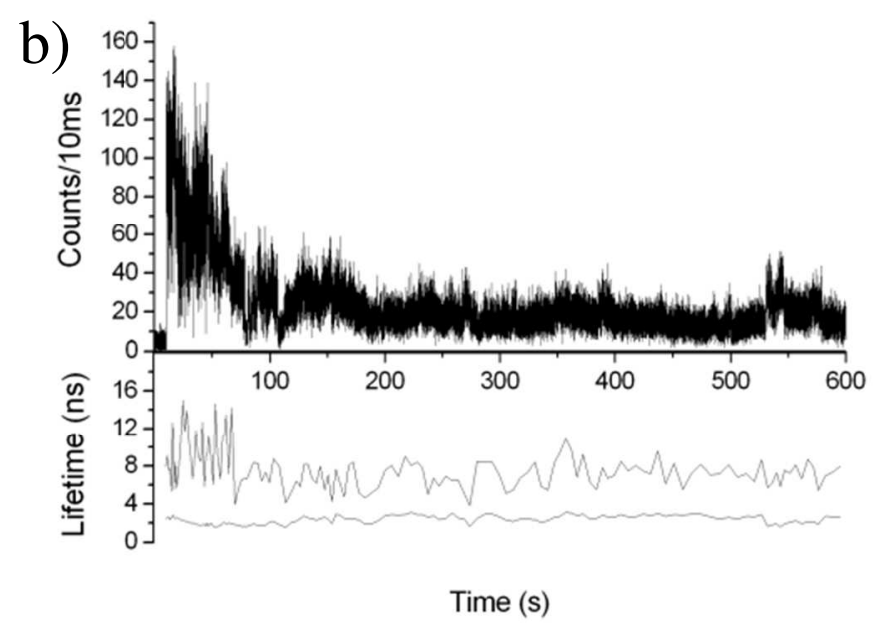

Figure 4. Fluorescence trajectories for two individual PFM-F8BT-PFM chains in PMMA showing a) mono-exponential and b) bi-exponential fluorescence decay kinetics. For each molecule, the upper panel shows counts with time in $10 \mathrm{~ms}$ bins and the lower panel shows the fluorescence lifetime(s) calculated every 10000 photons along the course of the trajectory.

Histograms showing the distributions of fluorescence lifetimes for F8BT and PFM-F8BT-PFM are shown in Figure 5. For histogram construction, a representative value for the lifetime(s) of each molecule was determined by averaging over the entire trajectory. For most molecules, the fluorescence lifetimes did not vary substantially over the course of the molecule's trajectory. This is particularly so for molecules showing mono-exponential behavior, i.e. all F8BT molecules and approximately one half 
of the PFM-F8BT-PFM molecules, so a representative value for the lifetime was straightforward to estimate and enter into the histograms in Figure 5a and b. The histograms in Figure 5c are built from molecules of PFM-F8BT-PFM showing bi-exponential kinetics with one histogram for each lifetime. The 12 molecules noted above that showed bi-exponential behavior for only a portion of their trajectory were included in the histograms in Figure 5c.

a)

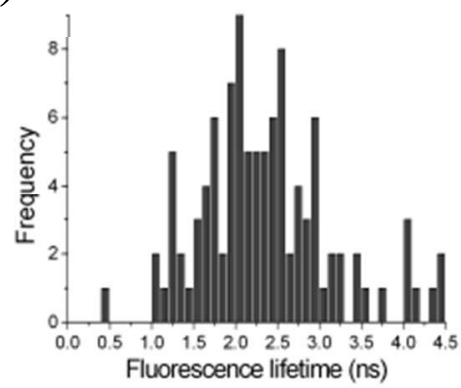

Fluorescence lifetime (ns)

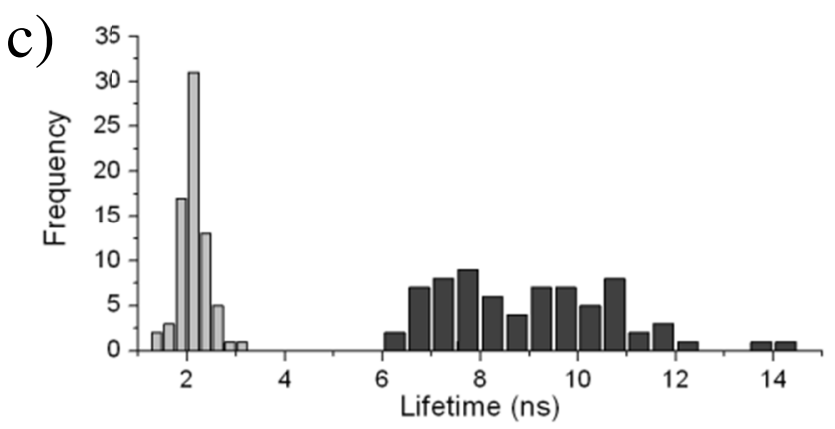

b)

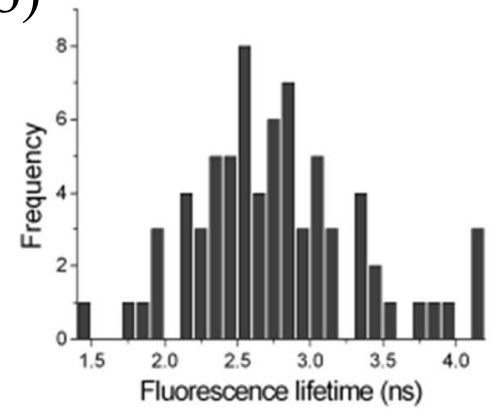

Figure 5. Distribution of fluorescence lifetimes from single chains in PMMA of a) F8BT, b) PFMF8BT-PFM showing mono-exponential decay kinetics and c) PFM-F8BT-PFM showing bi-exponential decay kinetics. The two histograms are of the two lifetimes extracted from bi-exponential fitting functions

Figure 5a shows the distribution of lifetimes for F8BT and is centered around $2.3 \mathrm{~ns}$. For the chains of PFM-F8BT-PFM that show mono-exponential decay, it is centered around $2.6 \mathrm{~ns}$. These values are very similar to the values obtained in toluene solution for these compounds. The other half of the PFMF8BT-PFM chains measured show two distinct lifetimes; a shorter lifetime distributed quite tightly 
around $2.1 \mathrm{~ns}$, and a longer lived component with more widely varying lifetime in the range of $\sim 6-12$ ns.

The observation of bi-exponential decay kinetics from single chains of the copolymer is unusual since single molecules are expected to show a single emission pathway, and therefore a mono-exponential decay. Two possible trivial explanations are poor dilution or contamination with another species resulting in two molecules within the confocal detection volume. These possibilities can be ruled out, since half of the measured molecules show bi-exponential decay kinetics, and no molecules have been recorded showing only a single, longer fluorescence lifetime. Another possibility is that since these molecules are $\sim 30 \mathrm{kDa}$ polymer chains, bi-exponential kinetics could arise from there being multiple emitting sites within a single chain. Defocused widefield imaging of single chains of the PFM-F8BT triblock copolymer in PMMA (Figure 6), reveals emission patterns consistent with there being a single emitting site within each polymer chain for 93\% of chains imaged (167 out of 179) ruling out multiple emission sites as the origin of bi-exponential kinetics observed in over $50 \%$ of single molecules studied by confocal microscopy. 
a)

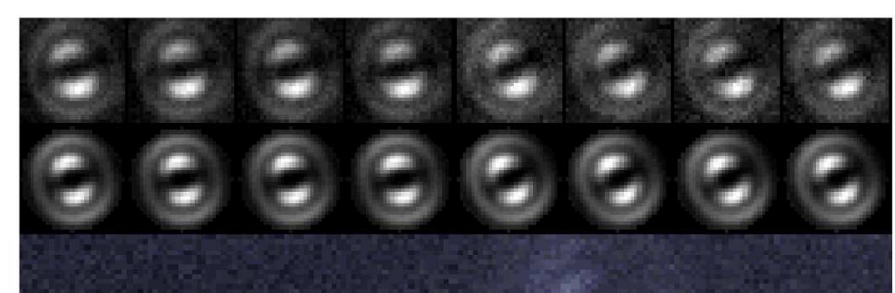

b)

Figure 6. a) Defocussed widefield images (upper panels) of a single PFM-F8BT-PFM molecule in PMMA with fitted patterns (lower panels) indicative of a single emitter. b) Defocussed wide field image of 4 single molecules of PFM-F8BT in PMMA with the molecule examined in a) indicated. Integration time 5 seconds, excitation $470 \mathrm{~nm}$.

A more likely explanation for the observed bi-exponential kinetics in single chains of PFM-F8BTPFM, consistent with the ensemble solution and neat film results, is that the two fluorescence lifetimes in some chains arise from delayed fluorescence due to charge transfer at the donor:acceptor interface. Delayed fluorescence arising from an LE - CT equilibrium is relatively unusual and has been reported at the single molecule level in only a few cases. ${ }^{15,40}$ To our knowledge, this is the first example of delayed fluorescence in single chains of a donor:acceptor conjugated copolymer arising from this mechanism. 
This interpretation is supported by concurrently recorded emission spectra (Figure 7) from single chains of PFM-F8BT-PFM. Half of the emitted fluorescence was directed to a CCD camera and an emission spectrum recorded every 5 seconds. There is no red-shifting of the emission in PFM-F8BTPFM compared to F8BT, i.e. F8BT-like spectra were recorded for all 149 PFM-F8BT-PFM molecules studied whether they showed mono- or bi-exponential decay kinetics. Although it is known that these polymers form exciplexes in the bulk state and some emission consistent with this was observed from a neat film of the copolymer, it is unlikely that these are formed at the single molecule level. Intrachain exciplexes with the required overlapped geometry would require stacking of the donor and acceptor blocks via chain folding. However these block copolymers are rigid, making this degree of chain collapse unlikely.

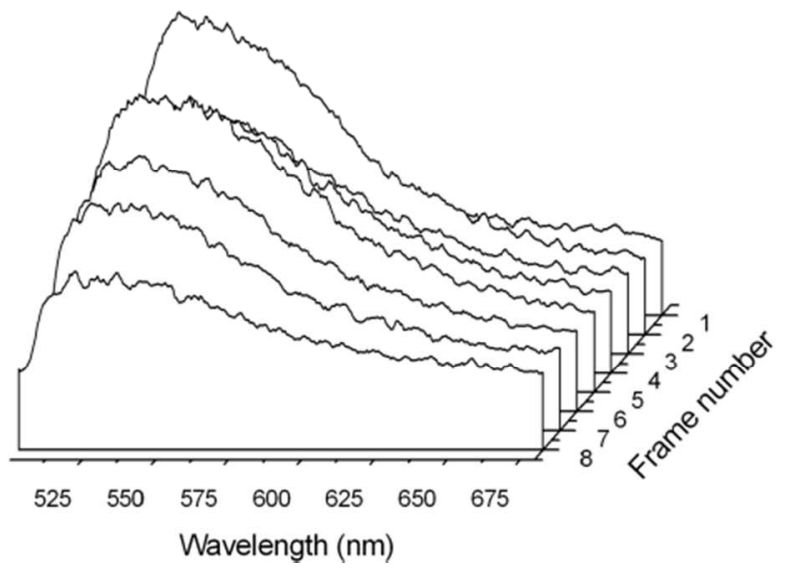

Figure 7. Emission spectra (integration time 5 seconds / spectrum) collected from a single, isolated chain of PFM-F8BT-PFM in a PMMA film. The molecule studied here displayed bi-exponential decay kinetics.

Using the same procedure adopted for bulk solution and neat film data, the rate constants and kinetic parameters for CT can be calculated from the time-resolved single molecule data. Taking representative values for decay constants and pre-exponential factors from the bi-exponential single molecule 
trajectory shown in Figure $4 \mathrm{~b}$, the forward and reverse rate constants $\left(k_{\mathrm{fwd}}\right.$ and $\left.k_{\mathrm{rev}}\right)$ were found to be 7.3 $\times 10^{7} \mathrm{~s}^{-1}$ and $1.5 \times 10^{8} \mathrm{~s}^{-1}$, respectively, with a non-radiative rate constant for recombination from the CT state to the GS $\left(k_{\mathrm{CR}}\right)$ of $2 \times 10^{5} \mathrm{~s}^{-1}$. This process is not competitive with the forward and reverse rate constants for the majority of the molecules measured, indicating that few excitations are lost through charge recombination from the charge transfer state back to the ground state. The equilibrium constant $\left(K_{\mathrm{eq}}\right)$ of 0.50 , and driving force $(\Delta \mathrm{G})$ of $0.018 \mathrm{eV}$ are comparable with the values calculated from the solution and neat film samples. For the molecules which show only mono-exponential decay kinetics, it may be that, due to local environment and chain conformation differences, the charge-transfer state energy is too high and forward CT is non-competitive with other radiative and non-radiative relaxation processes. 

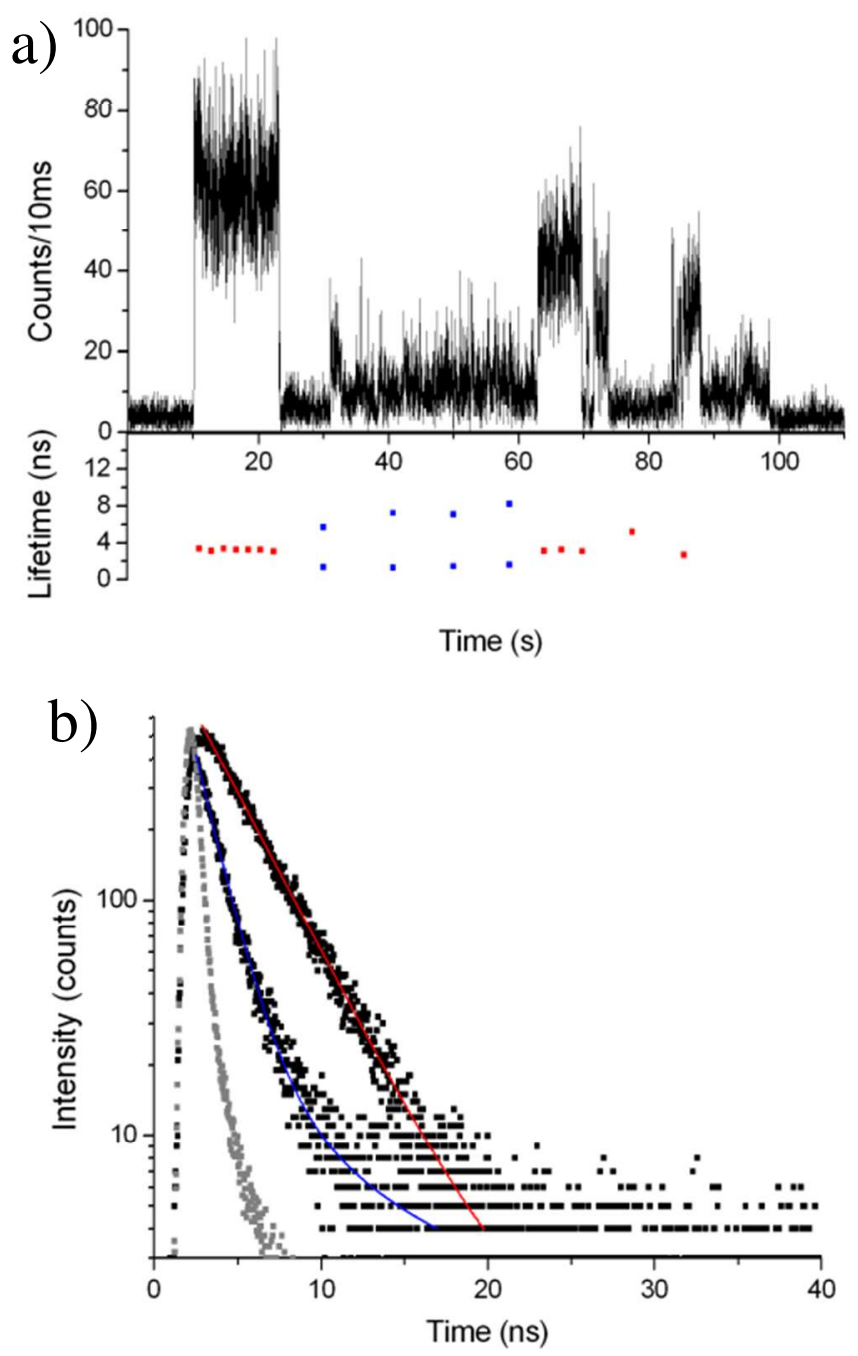

Figure 8. a) Fluorescence trajectory of an isolated PFM-F8BT-PFM chain in PMMA showing a change from single to bi-exponential fluorescence decay and b) example fluorescence decay profiles constructed from photons in the $15-18 \mathrm{~s}$ range and fitted by a single exponential decay (red) and from photons in the $40-50 \mathrm{~s}$ range and fitted by a bi-exponential decay (blue).

The distribution of SM behaviors is likely a result of the energy of the charge-transfer state being dependent on the local environment and polarity in the film. Furthermore, this raises the possibility that the local environment can fluctuate over time as well. Evidence for this can be seen in changes in the behavior of a single molecule over time, such as the one shown in Figure 8. This molecule shows 
evidence of both mono- and bi-exponential decays. At first, the CT state is unavailable and emission intensity (Figure 8a) is high and the kinetics are mono-exponential with a lifetime (Figure 8b) of $3.2 \mathrm{~ns}$. From 23 to 63 seconds, the intensity is much reduced and the decay kinetics become bi-exponential (with lifetime of 1.4 and $7.1 \mathrm{~ns}$ ) indicating that the CT state can now be accessed following excitation. This situation endures for $\sim 30$ seconds after which the CT state becomes unavailable and the decay becomes mono-exponential again. For molecules in a PMMA host matrix, polymer segmental motion can lead to changes in the local polarity that they experience. ${ }^{41}$ As the energy of the CT state is affected by polarity, this may lead to the fluctuating availability of the CT state and hence the appearance of delayed fluorescence.

\section{Conclusion:}

Donor-acceptor conjugated block copolymers are potentially useful materials for photovoltaic devices. In neat films, PFM-F8BT-PFM shows a broad, red-shifted emission typical of exciplexes, associated with a long fluorescence decay component. This exciplex emission is not found in solution or at the single molecule level in solid films, although complex fluorescence decay dynamics remain. A delayed fluorescence is observed under these conditions that is attributed to arise from a charge-transfer state that can form at the interface between the donor and acceptor. This type of delayed fluorescence, involving an equilibrium between the locally excited state and charge transfer state is unusual, and observed here even at the single chain level, highlighting the intrachain nature of the process. These results provide further insight into the complex photophysics that occurs in conjugated polymers and that is relevant to their applications as active materials in opto-electronic devices.

\section{Acknowledgement:}

We thank the Australian Research Council (ARC) for financial support of this research through grants DP0986166, LE100100131 and LE110100161. ENH acknowledges an Australian Postgraduate Award. 
We acknowledge Sam Ashworth for technical assistance with data collection. NCG is grateful to the School of Chemistry, The University of Melbourne for a Wilsmore Fellowship, and to Queen's College, Melbourne for a Sugden Fellowship.

\section{Supporting Information:}

Additional steady state spectra and Lippert-Mataga plots for PFM, F8BT and PFM-F8BT-PFM; fluorescence quantum yields of PFM-F8BT; example SM trajectory and lifetimes plot for a F8BT molecule; kinetic equations for delayed fluorescence. This material is available free of charge via the Internet at http://pubs.acs.org. 


\section{References:}

(1) Grimsdale, A. C.; Chan, K. L.; Martin, R. E.; Jokisz, P. G.; Holmes, A. B. Chem. Rev. 2009, 109, 897-1091.

(2) Banal, J. L.; Subbiah, J.; Graham, H.; Lee, J.-K.; Ghiggino, K. P.; Wong, W. W. H. Polym. Chem. 2013, 4, 1077-1083.

(3) Friend, R. H.; Gymer, R. W.; Holmes, A. B.; Burroughes, J. H.; Marks, R. N.; Taliani, C.; Bradley, D. D. C.; Dos Santos, D. A.; Bredas, J. L.; Logdlund, M.; Salaneck, W. R. Nature 1999, 397, 121-128.

(4) Kippelen, B.; Brédas, J. L. Energy Environ. Sci. 2009, 2, 251-261.

(5) Li, C.; Liu, M.; Pschirer, N. G.; Baumgarten, M.; Müllen, K. Chem. Rev. 2010, 110, 6817-6855.

(6) Arias, A. C.; MacKenzie, J. D.; Stevenson, R.; Halls, J. J. M.; Inbasekaran, M.; Woo, E. P.; Richards, D.; Friend, R. H. Macromolecules 2001, 34, 6005-6013.

(7) Hoppe, H.; Sariciftci, N. S. J. Mater. Chem. 2006, 16, 45-61.

(8) Mulherin, R. C.; Jung, S.; Huettner, S.; Johnson, K.; Kohn, P.; Sommer, M.; Allard, S.; Scherf, U.; Greenham, N. C. Nano Lett. 2011, 11, 4846-4851.

(9) Bicciocchi, E.; Chen, M.; Rizzardo, E.; Ghiggino, K. P. Polym. Chem. 2012, 4, 53-56.

(10) Guo, C.; Lin, Y.-H.; Witman, M. D.; Smith, K. A.; Wang, C.; Hexemer, A.; Strzalka, J.; Gomez, E. D.; Verduzco, R. Nano Lett. 2013, 13, 2957-2963.

(11) Sommer, M.; Huettner, S.; Thelakkat, M. J. Mater. Chem. 2010, 20, 10788-10797.

(12) Morteani, A. C.; Sreearunothai, P.; Herz, L. M.; Friend, R. H.; Silva, C. Phys. Rev. Letts. 2004, $92,247402$.

(13) Morteani, A. C.; Dhoot, A. S.; Kim, J.-S.; Silva, C.; Greenham, N. C.; Murphy, C.; Moons, E.; Ciná, S.; Burroughes, J. H.; Friend, R. H. Adv. Mater. 2003, 15, 1708-1712.

(14) Higginbotham, H. F.; Cox, R. P.; Sandanayake, S.; Graystone, B. A.; Langford, S. J.; Bell, T. D. M. Chem. Commun. 2013, 49, 5061-5063.

(15) Bell, T. D. M.; Stefan, A.; Masuo, S.; Vosch, T.; Lor, M.; Cotlet, M.; Hofkens, J.; Bernhardt, S.; Muellen, K.; Van der Auweraer, M.; Verhoeven, J. W.; De Schryver, F. C. ChemPhysChem 2005, 6, 942-948.

(16) Barbara, P. F.; Meyer, T. J.; Ratner, M. A. J. Phys. Chem. 1996, 3654, 13148-13168.

(17) Redecker, M.; Bradley, D. D. C.; Inbasekaran, M.; Wu, W. W.; Woo, E. P. Adv. Mater. 1999, 11, 241-246.

(18) Petrozza, A.; Laquai, F.; Howard, I. A.; Kim, J.-S.; Friend, R. H. Phys. Rev. B 2010, 81, 205421. 
(19) Dias, F. B.; King, S.; Monkman, A. P.; Perepichka, I. I.; Kryuchkov, M. A.; Perepichka, I. F.; Bryce, M. R. J. Phys. Chem. B 2008, 112, 6557-6566.

(20) Yan, C.; Cadby, A.J.; Parnell, A.J. Tang, W.; Skoda, M. W. A.; Mohamad, D.; King, S. P.; Reynolds, L. X.; Haque, S. A.; Wang, T.; Parnell, S. R.; Holmes, A. B.; Jones, R. A. L.; Jones, D. J. J. Poly. Sci. Part B Polym. Phys. 2013, 51, 1705-1718.

(21) Holmes, A.; Jones, D.; Wong, W. H. W.; Haque, S. Conjugated block copolymers, methods of preparation and their use in heterojunction devices. PCT Int. Appl. (2010), WO 2010060159 A1 20100603.

(22) Kellogg, R. E.; Bennett, R. G. J. Chem. Phys 1964, 41, 3042-3045.

(23) Cox, R. P.; Higginbotham, H. F.; Graystone, B. A.; Sandanayake, S.; Langford, S. J.; Bell, T. D. M. Chem. Phys. Lett. 2012, 521, 59-63.

(24) Gomez, D. E.; Tan, T. A. T.; White, J. M.; Bell, T. D. M.; Ghiggino, K. P. Aust. J. Chem 2009, $62,1577-1582$.

(25) Bell, T. D. M.; Yap, S.; Jani, C. H.; Bhosale, S. V.; Hofkens, J.; De Schryver, F. C.; Langford, S. J.; Ghiggino, K. P. Chem. Asian J. 2009, 4, 1542-1550.

(26) Hooley, E. N.; Tilley, A. J.; White, J. M.; Ghiggino, K. P.; Bell, T. D. M. Phys. Chem. Chem. Phys., 2014, 16, 7108-7114.

(27) Patra, D.; Gregor, I.; Enderlein, J. J. Phys. Chem. A, 2004, 108, 6836-6841

(28) Böhmer, M.; Enderlein, J. J. Opt. Soc. Am. B, 2003, 20, 554-559

(29) Dedecker, P.; Muls, B.; Deres, A.; Uji-i, H.; Hotta, J.; Sliwa, M.; Soumillion, J.; Müllen, K.; Enderlein, J.; Hofkens, J. Adv. Mater., 2009, 21, 1079-1090

(30) Uji-I, H.; Melnikov, S. M.; Deres, A.; Bergamini, G.; De Schryver, F.; Herrmann, A.; Müllen, K.; Enderlein, J.; Hofkens, J. Polymer, 2006, 47, 2511-2518

(31) Bittner, E. R.; Ramon, J. G. S.; Karabunarliev, S. J. Chem. Phys 2005, 122, 214719.

(32) Stevens, M. A.; Silva, C.; Russell, D. M.; Friend, R. H. Phys. Rev. B 2001, 63, 165213.

(33) Palilis, L. C.; Lidzey, D. G.; Redecker, M.; Bradley, D. D. C.; Inbasekaran, M.; Woo, E. P.; Wu, W. W. Synth. Met. 2001, 121, 1729-1730.

(34) Wu, W. C.; Liu, C. L.; Chen, W. C. Polymer 2006, 47, 527-538.

(35) Janssen, A. J.; Veldman, B. D.; Meskers, S. C. J. Adv. Funct. Mater. 2009, 19, 1939-1948.

(36) Huang, Y.-S.; Westenhoff, S.; Avilov, I.; Sreearunothai, P.; Hodgkiss, J. M.; Deleener, C.; Friend, R. H.; Beljonne, D. Nat. Mater. 2008, 7, 483-489.

(37) Ramon, J. G. S.; Bittner, E. R. J. Phys. Chem. B 2006, 110, 21001-21009. 
(38) Gelinas, S.; Pare, O.; Nadeau-Brosseau, C.; Albert-Seifried, S.; Mcneill, C. R.; Kirov, K. R.; Howard, I. A.; Leonelli, R.; Friend, R. H.; Silva, C. J. Phys. Chem. C 2011, 115, 7114-7119.

(39) Gronheid, R.; Stefan, A.; Cotlet, M.; Hofkens, J.; Qu, J.; Müllen, K.; Van der Auweraer, M.; Verhoeven, J. W.; De Schryver, F. C. Angew. Chem. Int. Ed. 2003, 42, 4209-4214.

(40) Cotlet, M.; Masuo, S.; Luo, G.; Hofkens, J.; Van der Auweraer, M.; Verhoeven, J.; Müllen, K.; Xie, X. S.; De Schryver, F. C. Proc. Natl. Acad. Sci. 2004, 101, 14343-14348.

(41) Izquierdo, M. A.; Bell, T. D. M.; Habuchi, S.; Fron, E.; Pilot, R.; Vosch, T.; De Feyter, S.; Verhoeven, J.; Jacob, J.; Müllen, K.; Hofkens, J.; De Schryver, F. C. Chem. Phys. Lett. 2005, 401, 503-508. 
Table of Contents Figure:

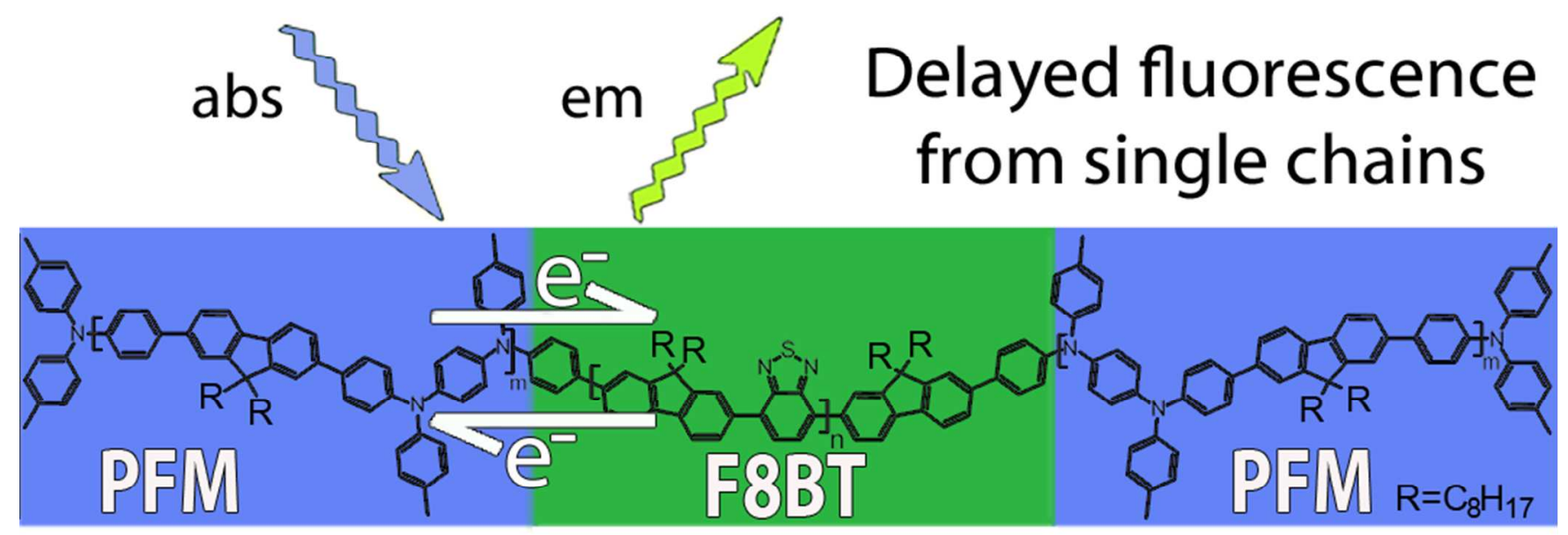

